\section{MONTAGU COTTAGE HOSPITAL, MEXBOROUGH.}

A CASE OF PYLORIC STENOSIS AND GASTRIC DILATATION OF VERY LONG DURATION (21 YEARS); POSTERIOR GASTRO-ENTEROSTOMY ; COMPLETE RECOVERY.

(Under the care of Mr. B. Crossfield Stevens.)

THE patient was a man, aged 40 years. He was thin, emaciated, and bedridden, and unable to retain food or to digest it. He stated that his illness dated from the age of 18 years, when a horse rolled on him. Pain in the stomach and vomiting came on soon afterwards and three months later he went to the Sheffield Infirmary, where he was put to bed, kept on a milk diet, and had his stomach washed out. He still vomited and had pain a few hours after food. After three months as an in-patient he was made an out-patient and continued to attend for three years and was able to work occasionally. Ten years previously, still suffering from the same symptoms, he went to the Sheffield Royal Hospital and after five weeks' lavage, dieting, and rest was discharged. From that time up to two months previously, when first seen by $\mathrm{Mr}$. Stevens, he had been treated privately. When Mr. Stevens saw him the emaciation was extreme; the man was unable to take anything without pain accompanied by vomiting, proximate or remote, and was going downhill fast. The friends were very adverse to any surgical procedure until they understood that it was operation or a lingering death. Under carbonio acid gas distension the stomach was quite two inches below the umbilicus. The pylorus could not be felt but most of the pain was at the cardiac end. Succussion splash was easily obtained and was very pronounced. The vomit was acid and frothy ; it was never tinged with blood.

Owing to the weak condition of the patient he was given 20 minims of liquor strychninæ hypodermically and a saline enema with brandy and the operation was completed within three-quarters of an hour. The pylorus was a hard gristly mass but gave no indications of being malignant nor were there any adhesions to the gall-bladder or any viscus. The stomach having been washed out a few hours before was empty and large. The ordinary method of posterior gastroenterostomy was done as Mr. Stevens had seen no vomiting usually after this ronte. The transverse mesocolon was opened, the stomach was pulled through and clamped, and the jejunum was looped up and ligated with rubber tubing. The anastomosis was made over a bone bobbin to act as a temporary splint for the surfaces to join over. Celluloid thread was used for the serous sutures and catgut for the mucous ones.

The patient was rather collapsed on return to bed. Liquor strychninæ and brandy were given every four hours. Hot water and plasmon were given by the mouth. A little pain and distension were present on the second day and this was relieved by the flatus tube. After this the patient never had a bad symptom; the pulse always kept below 100 . The temperature was normal. There was never any hiccough or sickness. Six weeks after the operation the patient was well and walking about and was enjoying his food and had gained weight considerably. Great credit is due to the matron and the nurses for their assiduous attention.

\section{Itterited Soxicties.}

\section{MEDICAL SOCIETY OF LONDON.}

\section{The Treatment of Urinary Tuberculosis.}

A MeETing of this society was held on March 28th, Dr. F. DE Ha villand Hall, the President, being in the chair.

Mr. E. HURRY FENWICK read a paper on the Treatment of Early Urinary Iuberculosis. He advocated the use of Koch's new tuberculin in cases where the mucous membrane of the bladder was alone affected by the disease. He had used this remedy since its introduction in 1896 and found that in many instances it afforded real relief to the pain in the lower urinary passages. No claim for cure was advanced. It must not be used anless the tubercle bacilli were found in the urine by a competent observer. Its exhibition had to be carefully watched, for it was liable to light up trouble in one or other kidney latently affected by tuberculosis. In a large number of cases of urinary tuberculosis the cystoscope enabled the clinician to decide as to whether the kidney was affected or not, for the chronic form of the disease was almost always unilateral and the kidney was implicated early in the course of the disorder. When the kidney was shown by the cystoscope to have a ureter condensed and choked by peri-ureteritis he advocated its removal. He gave a list of 25 nephrectomies for tuberculosis with one death. Four of the female cases in this list became pregnant sub. sequently to the nephrectomy, two being delivered of healthy children. This afforded evidence that a single kidney in a tuberculous patient could bear the stress of pregnancy. $\mathrm{Mr}$. Fenwick considered that the heavy death.rate of nephrectomy for tubercle of the kidney, just as that for carcinoma of the kidney, depended upon the surgeon not operating early, and he contended that the cystoscope would enable earlier interference and more judicious after-treatment. Should the cystoscope show a single ulcer in the bladder (and that condition was a rare one) he advised that it should be cut away suprapubically instead of using tuberculin. The same lines were employed in the male affected by genito-urinary tuberculosis. The affected testis or testes, vasa and seminal vesicles must be sacrificed if any good was to be effected. Should, however, the deposits be many and widespread, or should the history of tuberculosis of parts other than the genito urinary tract be obtainable, operation and tuberculin were useless and the best that could be done consisted in hygienic, dietetic, and therapeutic measures.

Dr. A. E. WRIGHT said that he had been engaged on work on somewhat similar lines. The use that had been made with the new tuberculin was not great. The new tuberculin consisted of the protoplasm of the tubercle bacilli and was a "vaccine" against tuberculosis similar to the "vaccine" against plague and, as he had more recently demonstrated, against streptococcic infection. The new tuberculin could be used with success in those cases in which the tubercle was local and he had used it in such cases with a fair measure of success. He had inoculated a considerable number of cases and from the examination of the blood he had been enabled to construct a curve showing the increase of immunity. He had, however, only treated one case of urinary tuberculosis but in that case very marked improvement had taken place. He thought that it was only by an examination of the blood that a true estimate of the effect produced by the inoculation could be obtained. After the frst inoculation a negative phase was produced which after two or three days became a positive phase. The positive phase gradually fell away and then it was that a fresh inoculation should take place. Immunity was in this way built up and he had treated several of the severer forms of tuberculosis in this manner with success.

Mr. F. SWINFORD EDWARDS said that tuberculosis of the urinary passages was one of the most distressing affections He had treated a considerable number of such cases with Koch's new tuberculin and in all of them the symptoms were much relieved and three cases might be said to have been cured. This form of treatment marked a great advance. He had never knowingly tried it in tuberculous disease of the kidney. He thought that it might be of value in diagnosis as it gave rise to pain in the affected kidney. $\mathrm{He}$ asked why Mr. Fenwick did not recommend it in single ulcer of the bladder.

Mr. J. G. PARDOE said that he was quite certain that tuberculin injections were not only useless but actually harmful in advanced disease of the kidney. He had used it in six cases with unsatisfactory results. He asked whether it was possible to distinguish between an ascending or a descending tuberculosis of the ureter by the nature of the local affection of the bladder in the neighbourhood of the areter. He said that a solitary tuberculous ulcer was most frequently situated on the trigone of the bladder and that it would be difficult to remove it from this situation.

Mr. J. W. Thomson WALKER said that he had treated some eight cases of tubercle of the bladder by inoculation and had had three cases under observation for from three to ten months. In only one of these cases was there no improvement while in all the rest there was very marked improvement The inoculation also tended to diminish hæmorrhage. In three cases of kidney tubercle and in one of ascending tubercle of the ureter no improvement had taken place. In two cases of early primary tubercle of the kidney 